\title{
A single institutional retrospective evaluation for younger patients with primary central nervous lymphomas on a modified R-MPV regimen followed by radiotherapy and high dose cytarabine
}

\author{
Keiichiro Hattori, ${ }^{1)}$ Mamiko Sakata-Yanagimoto, ${ }^{1)}$ Yasushi Okoshi, ${ }^{2)}$ Takayasu Kato, \\ Naoki Kurita, ${ }^{1)}$ Yasuhisa Yokoyama, ${ }^{1)}$ Naoshi Obara, ${ }^{1)}$ Shingo Takano, ${ }^{3)}$ Eiichi Ishikawa, \\ Tetsuya Yamamoto, ${ }^{3)}$ Akira Matsumura, ${ }^{3)}$ Yuichi Hasegawa, ${ }^{1)}$ Shigeru Chiba ${ }^{1)}$
}

\begin{abstract}
We conducted a retrospective analysis of patients younger than 60 years $(N=10$, median age 54.5) with newly diagnosed primary central nervous system lymphoma (PCNSL) at the University of Tsukuba Hospital from January 2008 to November 2016. All the patients were scheduled to receive a single regimen without registration to any clinical trials. This was based on a phase 2 study by Memorial Sloan-Kettering Cancer Center (MSKCC); induction chemotherapy with rituximab, methotrexate, procarbazine, and vincristine (R-MPV) (five to seven cycles), followed by whole-brain radiotherapy (rd-WBRT) (23.4 Gy) and two high-dose cytarabine (HD-AC) cycles as a consolidation. The median age was 54.5 years, and median follow up duration was 33.1 months. The 3-year overall survival (OS) and progression-free survival (PFS) were $69 \%$ (95\% CI 31-89\%) and 56\% (95\% CI 20-81\%). The median OS and PFS were not reached, respectively. Acute and delayed toxicities were manageable. In particular, OS and PFS of seven patients who achieved CR by the R-MPV induction chemotherapy were significantly superb (3-year OS, 100\%; 3-year PFS, 80\%), implying that a large proportion of patients in CR after the completion of this treatment may achieve durable disease control.

On the other hand, all of the three patients who had progressive disease during this treatment died of disease progression within 1 year after diagnosis without achieving CR. Identifying the patients having a risk of failure in the R-MPV induction chemotherapy is important, and may allow us to consider a potentially more effective regimen.
\end{abstract}

Keywords: R-MPV induction therapy followed by consolidation rdWBRT and HD-AC, primary central nervous system lymphoma, CR after completion of treatment

\section{INTRODUCTION}

Primary central nervous system lymphoma (PCNSL) is diffuse large B-cell lymphoma (DLBCL) that arises within the brain or eyes. It accounts for around $2-3 \%$ of primary malignant brain tumors and less than $1 \%$ of non-Hodgkin lymphomas (NHL) in adults. ${ }^{1}$ Historically, whole-brain radiotherapy (WBRT) was the sole treatment for PCNSL but the outcome of PCNSL treated with WBRT alone was not favorable. ${ }^{2}$

Combined modality therapy (CMT) using high-dose methotrexate (HD-MTX) and WBRT was first described in 2000 and since then has demonstrated improvement of response rate and prognosis. ${ }^{3}$ Now, there is consensus that CMT is superior to radiotherapy alone, although this was not confirmed in a randomized trial. ${ }^{3}$ As a result, HD-MTX in combination with WBRT has been chosen as the backbone in most of the currently used regimens. ${ }^{3}$ Although this CMT strategy results in high rates of disease remission, the majority of patients eventually progress and only a minority (20$40 \%$ ) are cured of their disease. Additionally problematic is the delayed neurotoxicity associated with CMT that is clinically evident in approximately one-third of patients, particularly in those at higher ages.

Recently, encouraging response rates have been achieved with rituximab-containing regimens in several clinical trials. , $^{4,6,6,7}$

\footnotetext{
Received: March 14, 2017. Revised: May 5, 2017. Accepted: May 15, 2017. J-STAGE Advance Published: August 4, 2017

${ }^{11}$ Department of Clinical and Experimental Hematology, Institute of Clinical Medicine, University of Tsukuba, Tsukuba, Japan, ${ }^{2}$ Ibaraki Clinical Education and Training Center, University of Tsukuba Hospital; Department of Hematology, Ibaraki Prefectural Central Hospital, Ibaraki, Japan, ${ }^{3}$ Department of Neurosurgery, Institute of Clinical Medicine, University of Tsukuba, Tsukuba, Japan.

Corresponding author: Shigeru Chiba, Department of hematology, Faculty of Medicine, University of Tsukuba, 1-1-1 Tennodai, Tsukuba, Ibaraki, 305-8575, Japan. E-mail: schiba-tky@umin.net
} 
In a study of the 90Y-labeled anti-CD20 antibody, single photon emission computed tomography (SPECT) imaging with ${ }^{111}$ In ibritumomab tiuxetan was performed in 6 patients before treatment with $90 \mathrm{Y}$ ibritumomab tiuxetan, and high uptake in the tumors in central nervous system was shown in 4 out of these 6 patients with PCNSL. ${ }^{9}$ This report suggests that rituximab may achieve therapeutic concentrations in regions of a brain tumor manifesting contrast enhancement secondary to blood brain barrier (BBB) disruption. ${ }^{9} \quad$ Ultimately, this may support the rationale for incorporation of rituximab into induction regimens for PCNSL

Among the rituximab containing regimens, the multicenter phase 2 study, which evaluated the efficacy of rituximab, methotrexate, procarbazine, and vincristine (R-MPV) followed by consolidation reduced-dose whole-brain radiotherapy (rdWBRT) and high-dose cytarabine (HD-AC) in PCNSL, demonstrated a high response rate (objective response rate (ORR), 95\%; 3-year overall survival (OS), $77 \%$; 3-year progression-free survival (PFS), 51\%), while the frequency of delayed neurotoxicity was low. ${ }^{4}$ However, reproducibility of this regimen has not been sufficiently confirmed, particularly in younger Japanese patients with PCNSL. Although efficacy of the R-MPV regimens in Japanese PCNSL patients was reported in a meeting abstract (Nakazaki K, et al., 2015; Harada Y, et al., 2013), any detailed information has not been published. ${ }^{10,11}$ We retrospectively analyzed the efficacy, acute toxicity, and delayed neurotoxicity of younger Japanese patients with PCNSL, treated by the R-MPV induction therapy, followed by reduced- or standard-dose WBRT (23Gy or $45 \mathrm{~Gy}$ ) and HD-AC consolidation at the University of Tsukuba Hospital in 2008-2016.

\section{MATERIALS AND METHODS Patients}

Between January 2008 and November 2016, a total of twelve consecutive patients with newly diagnosed PCNSL aged younger than 60 years, who were treated with the R-MPV induction therapy followed by consolidation rdWBRT and HD-AC at the University of Tsukuba Hospital, were identified from the medical records. ${ }^{4}$ Two patients were excluded from this analysis: one was initially infected with HIV and the other was treated with gamma-knife radiosurgery prior to diagnosis of PCNSL. The remaining ten patients were analyzed in a retrospective manner. Stereotactic surgery or tumor resection under craniotomy was performed for biopsy. Diagnosis was made on the basis of histological and immunohistochemical studies at the central pathological facility within the hospital. This retrospective study was approved by the institutional review board at the University of Tsukuba Hospital.

\section{Treatment and response evaluation}

We modified the original R-MPV regimen. The dose of rituximab in R-MPV was reduced from $500 \mathrm{mg} / \mathrm{m}^{2}$ to 375 $\mathrm{mg} / \mathrm{m}^{2}$. Treatment started with induction R-MPV chemotherapy ( 1 cycle=14 days), as follows: day 1 , rituximab 375 $\mathrm{mg} / \mathrm{m}^{2}$; day 2 , methotrexate $3.5 \mathrm{~g} / \mathrm{m}^{2}$ (over 2 hours), vincristine $1.4 \mathrm{mg} / \mathrm{m}^{2}$. Procarbazine was administered at $100 \mathrm{mg} /$ $\mathrm{m}^{2} / \mathrm{d}$ on days 1 through 7 (odd cycles only). ${ }^{4}$ Patients with evidence of lymphoma cells in cerebrospinal fluid (CSF) received $10 \mathrm{mg}$ of intraspinal MTX and cytarabine in each cycle. All patients who had grade 4 neutropenia received prophylactic lenograstim $(2 \mu \mathrm{g} / \mathrm{kg} / \mathrm{d}$, subcutaneously for 3 to 5 days) after each cycle.

Response was assessed using magnetic resonance imaging (MRI) with contrast enhancement according to the following criteria: complete response (CR) was determined by the absence of any contrast enhancement on the MRI of the brain, and partial response (PR) by at least a $50 \%$ reduction in the contrast-enhancing lesion seen on MRI as compared with baseline imaging. Patients were evaluated for response after five cycles of R-MPV. If CR was achieved, the patients went on to receive rd-WBRT (23.4 Gy fractionated at 1.8 Gy over 13 fractions) 3 to 5 weeks after completion of chemotherapy (Table 2, patient 5, 6, 8, and 10). If judged as a partial response, the patient received two additional cycles of R-MPV and was re-evaluated by MRI. If the patient achieved CR after seven cycles of induction chemotherapy, they then went on to receive rdWBRT (Table 2, patient 2). If small but persistent enhancing abnormalities, which were difficult to ascertain whether these represented residual niduses of tumor or scar tissue, were seen on MRI after 5 cycles of the R-MPV induction therapy, the patients also received two additional cycles of the R-MPV induction therapy and were re-evaluated. If the image of the abnormality did not change on MRI after the additional cycles of the R-MPV induction therapy, we categorized these as CR and rdWBRT was given to them (Table 2, patient 1,4). After radiotherapy, two courses of consolidation HD-AC were given at $3 \mathrm{~g} / \mathrm{m}^{2} / \mathrm{d}$ on

Table 1. Patient characteristics $(N=10)$

$\begin{array}{lc}\text { Age (years) } & 54.5 \\ \text { Median } & 47-59 \\ \text { Range } & \\ \text { KPS } & 70 \\ \text { Median } & 40-90 \\ \text { Range } & \\ \text { Sex } & 4 \\ \text { Male } & 6 \\ \text { Female } & \\ \text { Histology } & \\ \text { DLBCL } & 10 \\ \text { Menigeal involvement } & 0 \\ \text { Ocular involvement } & 0\end{array}$

KPS Karnofsky performance score $D L B C L$ diffuse large B cell lymphoma 
days 1 and 2 of each cycle (d1-28). Prophylactic lenograstim was given after each cycle.

Routine supportive treatments were given. In short, patients with normal cardiac and renal function were given 2-3 1 of hydration per day during HD-MTX. Urine alkalization with sodium bicarbonate was started 1 day before the administration of MTX. Urine $\mathrm{pH}$ was monitored 3 times a day during MTX administration. These supportive treatments were continued until the serum MTX concentration decreased to below $0.1 \mathrm{~mol} / \mathrm{l}$.

Three patients had progressive disease after cycle 1 (patient 3), 3 (patient 9), and 5 (patient 7) of the R-MPV induction therapy. Then, the induction therapy was stopped, and salvage WBRT or palliative therapy was selected for these patients.

\section{Follow-up and assessment}

All these evaluations were performed by referring to clinical records. In the survival analysis, OS was defined as "survival without death as a result of any cause" and PFS as "survival without disease progression or death as a result of PCNSL". In each survivor, day 0 was defined as the day on which the induction therapy was initiated. Acute and delayed toxicities were evaluated according to the National Cancer Institute's Common Terminology Criteria for Adverse Events (CTCAE) (version 4; http://evs.nci.nih.gov/ftp1/ CTCAE/About.html).

Kaplan-Meier survival curves were analyzed with the log rank test using the EZR. ${ }^{12}$

\section{RESULTS \\ Patient characteristics}

The characteristics and outcomes of the ten patients with newly diagnosed PCNSL are summarized in Tables 1 and 2 . The median age was 54.5 years, and median follow up duration was 33.1 months. No patients had ocular or meningeal involvement. In total, all ten patients were assessed for response to induction chemotherapy with R-MPV.

In three patients, the period between the first visit to a physician because of symptoms related to PCNSL and treatment was remarkably longer than that of other patients (Table 2; patient 3, 5, and 9). Patients 3 and 5 were initially diagnosed as having multiple sclerosis and treated with several courses of corticosteroid pulse therapy, and were subsequently diagnosed as having PCNSL. As a result, start of therapy was postponed. The induction therapy of patient 9 was postponed due to infection that was suspected to be associated with meningitis.

\section{Response and survival}

Four patients (40\%) (patients 5, 6, 8, and 10) achieved $\mathrm{CR}$ by the fifth cycle of the R-MPV induction therapy (Table 2 ). One patient (patient 2) with a PR after the fifth cycle received the additional two cycles of the induction therapy and then achieved CR on MRI (Table 2). The other two patients (patients 1 and 4) who had minimal abnormalities at the post cycle 5 evaluation also received two additional cycles of the R-MPV induction therapy (Table 2). The images did not change after the additional cycles in both cases, and thus, we categorized these abnormalities as $\mathrm{CR}$ (Table 2). All these seven patients maintained CR at the

Table 2. Patients and outcomes

\begin{tabular}{|c|c|c|c|c|c|c|c|c|c|c|c|}
\hline UPN & Age & Sex & $\begin{array}{l}\text { The period } \\
\text { between the first } \\
\text { examination and } \\
\text { treatment }\end{array}$ & $\begin{array}{l}\text { Completion of } \\
\text { the induction } \\
\text { therapy } \\
(5 \times \mathrm{R}-\mathrm{MPV})\end{array}$ & $\begin{array}{l}\text { Evaluation } \\
\text { after } 5 \times \\
\text { R-MPV }\end{array}$ & $\begin{array}{l}\text { Additional } \\
\text { R-MPV }\end{array}$ & $\begin{array}{l}\text { Evaluation } \\
\text { after } \\
\text { additional } \\
\text { R-MPV }\end{array}$ & Radiation & $\begin{array}{l}\text { Evaluation } \\
\text { After radiation } \\
\text { therapy }\end{array}$ & $\begin{array}{c}\text { OS } \\
\text { (day) }\end{array}$ & $\begin{array}{l}\text { PFS } \\
\text { (day) }\end{array}$ \\
\hline 1 & 58 & $\mathrm{~F}$ & 18 & Yes & $\mathrm{CR}^{* 1}$ & Yes $(2) * 2$ & $\mathrm{CR}$ & rdWBRT & CR & $1406+$ & $1406+$ \\
\hline 2 & 49 & $\mathrm{~F}$ & 27 & Yes & PR & Yes $(2) * 2$ & $\mathrm{CR}$ & rdWBRT & $\mathrm{CR}$ & $2246+$ & $2246+$ \\
\hline 3 & 59 & $\mathrm{~F}$ & 251 & No & NA & NA & NA & NA & NA & 13 & 13 \\
\hline 4 & 51 & $\mathrm{~F}$ & 29 & Yes & $\mathrm{CR} * 1$ & Yes $(2) * 2$ & $\mathrm{CR}$ & rdWBRT & CR & $2531+$ & $2531+$ \\
\hline 5 & 47 & M & 97 & Yes & $\mathrm{CR}$ & No & NA & rdWBRT & CR & 1333 & 823 \\
\hline 6 & 54 & $\mathrm{~F}$ & 35 & Yes & $\mathrm{CR}$ & No & NA & rdWBRT & CR & $1261+$ & $1261+$ \\
\hline 7 & 52 & $\mathrm{~F}$ & 12 & Yes & PD & No & NA & WBRT (36Gy) & PD & 278 & 116 \\
\hline 8 & 55 & M & 38 & Yes & $\mathrm{CR}$ & No & NA & rdWBRT & CR & $728+$ & $728+$ \\
\hline 9 & 58 & M & 72 & No & NA & NA & NA & WBRT(39.6Gy) & PD & 158 & 58 \\
\hline 10 & 58 & M & 27 & Yes & $\mathrm{CR}$ & No & NA & rdWBRT & CR & $255+$ & $255+$ \\
\hline
\end{tabular}

$U P N$ unique patient number, $P F S$ progression-free survival, $O S$ overall survival, $C R$ complete response, $P R$ partial response, $P D$ progression disease, $N A$ not available, WBRT whole brain radiotherapy, $r d$ reduced dose, "+" alive at the cutoff point

*1 Patients had minimal abnormalities, which were difficult to evaluate whether these were tumor or scar tissue,

*2 Patients received two additional cycles of the R-MPV induction therapy. 
completion of the R-MPV induction therapy. They received rdWBRT at $23.4 \mathrm{~Gy}$. After radiotherapy, these patients received two consolidation HD-AC therapies. As a result, six of these seven patients remain alive at the time of submission of this manuscript and are free of disease progression. In one male patient achieving CR (patient 5), lymphoma progressed at 22 months after completion of the therapy. Then, he received high-dose chemotherapy using etoposide, cytarabine, and melphalan (BEAM) with autologous stem cell transplant (ASCT), but he experienced disease relapse within six months and died 1 year after ASCT.

The other three patients (30\%) (patients 3, 7 and 9) did not achieve CR by the R-MPV induction therapy (Table 2). One patient (patient 7) with progressive disease after the induction therapy received WBRT at only 36 Gy because the patient refused further doses of radiation. After radiotherapy, the patient died because of disease progression. Two patients (patients 3 and 9) progressed before the completion of the R-MPV induction therapy. Patient 3 died because of early progression after the first cycle of the R-MPV induction therapy. Patient 9 had disease progression after the third cycle of the induction therapy and then received WBRT at 39.6 Gy as salvage therapy. This subject subsequently died of disease progression.

For all ten patients, 3-year PFS was 56\% (95\% CI $20-81 \%$ ), with the median PFS not reached (Figure). The 3 -year OS was $68.6 \%$ (95\% CI 31-89\%), with the median

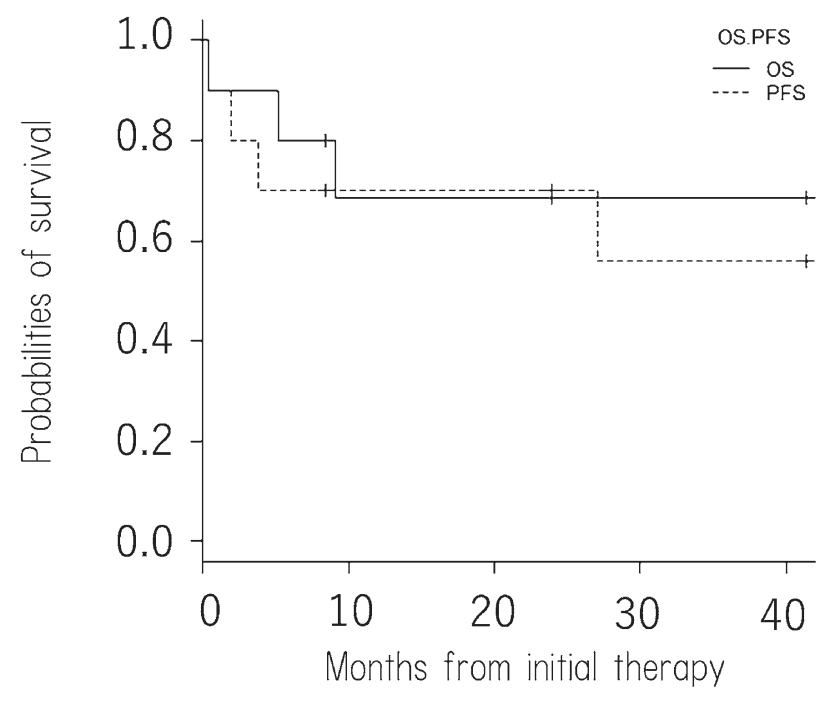

Fig. Overall survival (OS) and Progression free survival (PFS) after induction therapy.
OS not reached (Figure). OS and PFS of patients who achieved CR at the completion of the induction chemotherapy were favorable (3-year OS, 100\%; 3-year PFS, 80\%).

\section{Acute toxicity of induction chemotherapy}

The median number of R-MPV cycles was five (range, one to seven). The acute and severe toxicities that occurred during the induction therapy are summarized in Table 3. The most frequent toxicity was myelosuppression. Five patients $(50 \%)$ developed grade 3 or 4 neutropenia. Grade 3 anemia occurred in three patients $(30 \%)$. Two patients $(20 \%)$ had grade 3 aspartate transaminase (AST) and alanine transaminase (ALT) elevations, and one patient (10\%) had grade 3 gamma-glutamyl transpeptidase ( $\gamma$ GTP) elevation. These liver dysfunctions were recorded 3-9 days after the initiation of the induction therapy and were reversible without specific therapy. Three patients had febrile neutropenia (grade 3) during myelosuppression, which was managed and cured with antibiotics. One patient (patient 6) $(10 \%)$ had cognitive disturbance and gait disorder soon after completion of all treatment without lymphoma progression, which were reversible.

\section{DISCUSSION}

Several studies on PCNSL treated with immunochemotherapy in combination with WBRT have been reported. ${ }^{4,13-16}$ Although there are limitations in comparison across different cohorts, the OS and PFS of patients in this cohort are excellent (Figure, 3-year OS, 69\%; 3-year PFS, 56\%) and are comparable to those of the phase 2 study of MSKCC (3-year OS, 77\%: 3-year PFS, 51\%).

Unfortunately, three of ten patients (patients 3, 7, and 9) died of disease progression. Among these three patients, 2 patients (patients 3 and 9) had rapid progression of disease before the completion of the R-MPV induction therapy (Table 2). The initiation of induction therapies was delayed in these patients, which may partly account for the treatment failure. In general, histological diagnosis can be difficult at the early period of the clinical course if the lesion is located deep in the brain structure. Detection of somatic mutations in cell-free DNA (cfDNA), a recently developed method, may represent a minimally invasive tool helping for diagnosis. ${ }^{17}$

Several specific clinical parameters, such as MSKCC and IELSG prognostic score systems, were reported to predict the treatment outcome of PCNSL patients. ${ }^{18,19}$ We previously

Table 3. Severe acute toxicities during the induction therapy $(N=10)$

\begin{tabular}{ccccccc}
\hline Grade & Anemia & Thrombopenia & Neutropenia $^{\mathrm{a}}$ & Hepatitis & Renal toxicity & Cognitive disturbance \\
\hline 3 & $3(30)$ & 0 & $1(10)$ & $3(30)$ & 0 & $1(10)$ \\
4 & 0 & 0 & $4(40)$ & 0 & 0 & 0 \\
\hline
\end{tabular}

Patients` number and percentage are shown

${ }^{a}$ Three patients developed grade 3 febrile neutropenia 
found that MYD88 L265P mutations had a negative impact on the clinical outcome of elderly PCNSL patients. ${ }^{20}$ The impact of MYD88 L265P mutation should be examined for younger patients treated with the R-MPV regimen followed by consolidation rdWBRT and cytarabine.

Newly developed targeted drugs may provide treatment options in PCNSL patients. Ibrutinib, a selective covalent BTK inhibitor, is effective in patients with some B-cell malignancies by inhibiting the NF- $\mathrm{KB}$ pathway activity. ${ }^{21,22,23}$ The effectiveness of ibrutinib for CNS relapse of B-cell lymphomas was demonstrated. ${ }^{24}$ Recently, several studies have been conducted to show the effects on refractory/recurrent CNS lymphoma patients (NCT02203526, NCT02315326). ${ }^{25,26}$ Additionally, lenalidomide, an IMiD class biological agent, may also be effective for PCNSL via down-modulation of MUM1/IRF-4. ${ }^{27}$ A phase-2 trial combining rituximab and lenalidomide for recurrent PCNSL is ongoing (NCT01956695). ${ }^{28}$

In addition, there has been increasing interest in the role of high-dose chemotherapy (HDC) followed by ASCT in PCNSL as a consolidation approach in first-line therapy. ${ }^{29}$ Antonio Omuro et al. previously conducted a single center phase 2 study in newly diagnosed PCNSL utilizing the R-MPV induction therapy and HD-AC, followed by consolidation HDC-ASCT with thiotepa, busulfan, and cyclophosphamide (TBC). ${ }^{30}$ In their series, excellent disease-free control and OS (3-year OS, 81\%; PFS, 79\%) were observed, with an acceptable toxicity profile and minimal neurotoxicity. ${ }^{30}$ Interestingly, a small phase 2 study suggested that HDC-ASCT could replace consolidation radiotherapy, with a 3-year OS of 77\%. ${ }^{31}$ Thus, HDC followed by ASCT may be proposed as an alternative consolidation therapy instead of radiotherapy in PCSNL, especially for patients who achieved $\mathrm{CR}$ after the induction phase.

In conclusion, a 3-year overall survival rate of $100 \%$ was achieved by the R-MPV regimen followed by consolidation rdWBRT and cytarabine in Japanese patients achieving CR at the completion of this treatment. The effectiveness of the treatment was an important factor contributing to these favorable results. Further study is needed to identify the factors that predict the tolerance and response before treatment. Therapy can potentially be revised or newly invented to a potentially more effective regimen in patients who are destined to respond poorly to the initial treatment.

\section{AUTHOR CONTRIBUTIONS}

K.H., M.S-Y., and S.C. designed and performed research, analyzed data, and wrote the paper; Y.O., T.K., N.K., Y.Y., N.O., and Y.H designed and performed research; S.T., E.I., T.Y., and A.M. provided patient data.

\section{CONFLICT OF INTEREST}

S.C. received research funds from the Mitsubishi Tanabe Pharma Corporation, which sells MTX in Japan, the Nippon Shinyaku Corporation, which sells $\mathrm{AraC}$, and the Chugai
Pharmaceutical Corporation which sells procarbazine and rituximab. No other research support was provided for this study.

\section{REFERENCES}

1 Kluin P, Deckert M, Ferry J, Swerdlow SH, Campo E, et al. : WHO classification of tumours of haematopoietic and lymphoid tissues. $4^{\text {th }}$ ed, Lyon, IARC, pp. 240-241, 2008

2 Nelson DF, Martz KL, Bonner H, Nelson JS, Newall J, et al. : Non-Hodgkin's lymphoma of the brain: can high dose, large volume radiation therapy improve survival? Report on a prospective trial by the Radiation Therapy Oncology Group (RTOG): RTOG 8315. Int J Radiat Oncol Biol Phys 23(1): 9-17, 1992

3 Prica A, Chan K, Cheung MC : Combined modality therapy versus chemotherapy alone as an induction regimen for primary central nervous system lymphoma: a decision analysis. Br J Haematol 158(5): 600-607, 2012

4 Morris PG, Correa DD, Yahalom J, Raizer JJ, Schiff D, et al. : Rituximab, methotrexate, procarbazine, and vincristine followed by consolidation reduced-dose whole-brain radiotherapy and cytarabine in newly diagnosed primary CNS lymphoma: final results and long-term outcome. J Clin Oncol 31(31): 39713979,2013

5 Holdhoff M, Ambady P, Abdelaziz A, Sarai G, Bonekamp D, et al. : High-dose methotrexate with or without rituximab in newly diagnosed primary CNS lymphoma. Neurology 83(3): 235-239, 2014

6 Shah GD, Yahalom J, Correa DD, Lai R K, Raizer JJ, et al. : Combined immunochemotherapy with reduced whole-brain radiotherapy for newly diagnosed primary CNS lymphoma. J Clin Oncol 25(30): 4730-4735, 2007

7 Fritsch K, Kasenda B, Hader C, Nikkhah G, Prinz M, et al. : Immunochemotherapy with rituximab, methotrexate, procarbazine, and lomustine for primary CNS lymphoma (PCNSL) in the elderly. Ann Oncol 22(9): 2080-2085, 2011

8 Batchelor T, Grossman SA, Mikkelsen T, Ye X, Desideri S, et al. : Rituximab monotherapy for patients with recurrent primary CNS lymphoma. Neurology 76(10): 929-930, 2011

9 Maza S, Kiewe P, Munz DL, Korfel A, Hamm B, et al. : First report on a prospective trial with yttrium-90-labeled ibritumomab tiuxetan (Zevalin) in primary CNS lymphoma. Neuro Oncol 11(4): 423-429, 2009

10 Nakazaki K, Nakamura F, Mukasa A, Tanaka S, Takayanagi S, et al. : R-MPV therapy followed by radiotherapy and high dose cytarabine is effective for CNS lymphoma. Rinsho Ketuseki 56(9): 1416, 2015

11 Harada Y, Aoki T, Matsubara E, Morishita T, Suzuki T, et al. : R-MPV followed by reduced whole-brain radiotherapy in newly diagnosed primary CNS lymphoma. Rinsho Ketsueki 54(9): 1123,2013

12 Kanda Y : Investigation of the freely available easy-to-use software 'EZR' for medical statistics. Bone Marrow Transplant 48(3): 452-458, 2013

13 Chamberlain MC, Johnston SK : High-dose methotrexate and rituximab with deferred radiotherapy for newly diagnosed 
primary B-cell CNS lymphoma. Neuro Oncol 12(7): 736-744, 2010

14 Wieduwilt MJ, Valles F, Issa S, Behler CM, Hwang J, et al. : Immunochemotherapy with intensive consolidation for primary CNS lymphoma: a pilot study and prognostic assessment by diffusion-weighted MRI. Clin Cancer Res 18(4): 1146-1155, 2012

15 Holdhoff M, Ambady P, Abdelaziz A, Sarai G, Bonekamp D, Blakeley J, Grossman SA, Ye X : High-dose methotrexate with or without rituximab in newly diagnosed primary CNS lymphoma. Neurology 83(3): 235-239, 2014

16 Lee SY, Okoshi Y, Kurita N, Seki M, Yokoyama Y, et al. : Prognosis factors in Japanese elderly patients with primary central nervous system lymphoma treated with a nonradiation, intermediate-dose methotrexate-containing regimen. Oncol Res Treat 37(7-8): 378-383, 2014

17 Maxime F, Florent M, lodie B, Pierre-Julien V, Philippe B, et al. : Somatic Mutations Detected in Plasma Cell-Free DNA By Targeted Sequencing: Assessment of Liquid Biopsy in Primary Central Nervous System Lymphoma. ASH 57th Annual Meeting abstract 332, 2015

18 Abrey LE, Ben-Porat L, Panageas K S, Yahalom J, Berkey B, et al. : Primary central nervous system lymphoma: the Memorial Sloan-Kettering Cancer Center prognostic model. J Clin Oncol 24(36): 5711-5715, 2006

19 Ferreri AJ, Blay JY, Reni M, Pasini F, Spina M, et al. : Prognostic scoring system for primary CNS lymphomas: the International Extranodal Lymphoma Study Group experience. J Clin Oncol 21(2): 266-272, 2003

20 Hattori K, Sakata-Yanagimoto M, Okoshi Y, Matsumura A, Chiba S, et al. : MYD88 (L265P) mutation is associated with an unfavourable outcome of primary central nervous system lymphoma. Br J Haematol 177(3): 492-494, 2017

21 Wilson WH, Young RM, Schmitz R, Yang Y, Pittaluga S, et al. : Targeting B cell receptor signaling with ibrutinib in diffuse large B cell lymphoma. Nat Med 21(8): 922-926, 2015

22 Treon SP, Tripsas CK, Meid K, Warren D, Varma G, et al. : Ibrutinib in previously treated Waldenstrom's macroglobulinemia. N Engl J Med 372(15): 1430-1440, 2015
23 Roskoski R Jr. : Ibrutinib inhibition of Bruton protein-tyrosine kinase (BTK) in the treatment of B cell neoplasms. Pharmacol Res 113(Pt A): 395-408, 2016

24 Sophie Bernard, Lauriane Goldwirt, Sandy Amorim, Pauline Brice, Josette Brière, et al. : Activity of ibrutinib in mantle cell lymphoma patients with central nervous system relapse. Blood 126(14): 1695-1698, 2015

25 Dunleavy K, Lai CE, Roschewski M, Brudno JN, Widemann B, et al. : Phase I Study of Dose-Adjusted-Teddi-R with Ibrutinib in Untreated and Relapsed/Refractory Primary CNS Lymphoma. ASH 57th Annual Meeting abstract 472, 2015

26 : Christian G, Thomas K, Abdel-Wahab O, Omuro AM, Ingo M, et al. : Phase I Study of Single Agent Ibrutinib in Recurrent/ Refractory Primary and Secondary CNS Lymphoma. ASH 57th Annual Meeting abstract 3960, 2015

27 Ponzoni M, Issa S, Batchelor TT, Rubenstein JL : Beyond highdose methotrexate and brain radiotherapy: novel targets and agents for primary CNS lymphoma. Ann Oncol 25(2): 316-322, 2014

28 Houillier C, Choquet S, Touitou V, Martin-Duverneuil N, Navarro $\mathrm{S}$, et al. : Lenalidomide monotherapy as salvage treatment for recurrent primary CNS lymphoma. Neurology 84(3): 325-326, 2015

29 Rubenstein JL, Gupta NK, Mannis GN, Lamarre AK, Treseler P : How I treat CNS lymphomas. Blood 122(14): 2318-2330, 2013

30 Omuro A, Correa DD, DeAngelis LM, Moskowitz CH, Matasar MJ, et al. : R-MPV followed by high-dose chemotherapy with $\mathrm{TBC}$ and autologous stem-cell transplant for newly diagnosed primary CNS lymphoma. Blood 125(9): 1403-1410, 2015

31 Illerhaus G, Muller F, Feuerhake F, Schafer AO, Ostertag C, et al. : High-dose chemotherapy and autologous stem-cell transplantation without consolidating radiotherapy as first-line treatment for primary lymphoma of the central nervous system. Haematologica 93(1): 147-148, 2008 